oncogenes, abbreviated to this day as c-oncs, that would function as cancer genes by increased dosage ${ }^{6}$. Hence the term oncogene activation ${ }^{6}$. But subsequent work, mostly by others, has shown that viral onc genes and cellular progenitors have different functions because they have different structures ${ }^{7-9}$. So far, not a single cellular progenitor of a viral onc gene has been proved to be a cancer gene, except if it had been altered ('corrupted') by recombination with a retrovirus $^{7-9}$. Yet it seems that the appeal of this hypothesis is the probable basis for the current Nobel prize.

All the points I make here had been made before the current controversy, first in Nature and later in other journals ${ }^{7-9}$ and have not so far been challenged.

Peter Duesberg

University of California, Berkeley,

Department of Molecular and

Cell Biology.

Stanley Hall,

Berkeley, California 94720, USA

1. Nature 342, 328 (1989)

. Stéhelin, D. etal. Nature 260, 170-173 (1976)

3. Scolnick, E.M. \& Parks, W.P. J. Virol. 13, 1211-1219 (1974).

4. Tsuchida, N., Gilden, R.V. \& Hatanaka, M. Proc. natn Acad. Sci. U.S.A. 71, 4503-4507 (1974).

5. Huebner, R.J., \& Todaro, G. Proc. natn. Acad. Sci. U.S.A. 64, 1087-1094 (1969).

6. Bishop, M. \& Varmus, H.E. in RNA Tumor Viruses, Weiss, R., Teich, N., Varmus, H. \& Coffin, J. (eds) 999-1108 (Cold Spring Harbor Laboratory, New York, 1982).

7. Duesberg. P. H. Nature 304, 219-226 (1983)

8. Duesberg, P.H. Proc. natn. Acad. Sci. U.S.A. 84, 2117 Duesberg,
(1987).

9. Duesberg, P.H., Goodrich, D. \& Zhou, R.-P. Ann. N.Y Acad. Sci. 567, 259-273 (1989)

10. Scolnick, E.M. et al. J. Virol. 12, 458-463 (1973).

\section{Motive power}

SIR-Virginia Trimble clearly establishes (Nature 342, 11; 1989) that graduates of more prestigious universities publish for a longer professional period than those of other institutions. No one will want to squabble with this finding. But the conclusion that she draws, that "it still pays to go to the most prestigious graduate institution", is based on an assumption that is congenial to a physicist used to classes of identical objects. It is the assumption that the samples of students in the two kinds of institutions are equivalent and that their lifelong behaviour is the result of their institutional association. This ignores, however, the fact that only one in seven or even one in 10 of the applicants to a prestigious institution is accepted, while in less prestigious institutions the selection is far less severe.

One of the criteria on which this drastic selection is based is an indication of high motivation of the applicant. This criterion is of great importance because many studies have shown that high motivation, all else being equal, is usually more important for high achievement than mere intelligence. In other words, there is much to indicate that the continuing high moti- vation of graduates from prestigious institutions is due to the fact that they represent a sample selected for high motivation.

This conclusion does not deny the additional factor of an intellectual climate in the prestigious institution that favours continuing productivity nor other aspects of the environment (such as lower teaching load) that also favour high productivity. Yet all the evidence indicates that continuing high motivation is not primarily the effect of institutional association.

ERNST MAYR

Museum of Comparative Zoology,

Harvard University,

Cambridge, Massachusetts 01138, USA

\section{Nonrandom uses}

SIR-The word "random" is sometimes used in Nature and other publications where another term such as "chaotic", "unpredictable", "uncertain", "arbitrary" or "undetermined" should be used. The use of "random" to describe a process, behaviour or physical system should be reserved for cases where an author can prove the system is random.

The primary definition of "random" is "proceeding, made, or occurring without definite aim, reason, or pattern"1. "Apparently random fluctuations" $"$ would be better expressed as "unpredictable fluctuations" as there are "structures" and "regularity".

When "random" is used to denote a selection process ${ }^{3}$ or simulation method, close attention must be paid to the implementation of the randomizing process. That the chaotic orbits of a three-body system would upon ejection of a member "randomize the spin orientation" ${ }^{4}$ is neither demonstrated in the paper nor an expected result of the ejection. Instead, the spin orientation is deterministic and yet unpredictable. The reason and pattern for the spin orientation are known; it is the limits of observation and the limits of computability that prohibit prediction.

In a statistical sense, "random" is "of or characterizing a process of selection in which each item of a set has an equal probability of being chosen ${ }^{1}$ and is properly used in Nature 342, 128 (1989) when speaking of a "random distribution of orientations" as a metric.

Even the use of "random" in statistical analysis and computer science must be viewed critically ${ }^{5}$. Computers are used to generate sequences representing random selections using deterministic algorithms. Some common computer randomization methods produce less than exemplary results. These algorithms produce reproducible sequences that have qualities that emulate "random" behaviour (if such a thing as random behaviour exists). These computer-generated sequences are better termed "pseudo-random".

If system history influences succeeding states, then we cannot consider it random merely because we do not know the aim, reason or pattern. In dynamics we must carefully consider evidence such as power spectral distribution, trajectory, degrees of freedom, rate of divergence and dimensionality when branding a system "chaotic". The same burden of proof should apply to those who report a system to be "random".

THOMAS L. OCHS

US Bureau of Mines,

1450 Queen Avenue, SW, Albany, Oregon 97321, USA

1. Random House Directory of the English Language 2nd Edn (1987)

2. James, I.N., \& James, P.M. Nature 342, 53 (1989).

3. Shulman, S. Nature 342, 4 (1989).

4. Ables, J.G. et al. Nature 342, 161 (1989)

5. Park, S. K. \& Miller, Keith W. Random Number Generators, Good Ones Are Hard To Find, Communications of the ACM 31, $1192-1201$ (1986)

\section{Embryo research}

SIR-Public attitudes to fertilization and embryology have been shown to be considerably pliable. This is well illustrated in the Warnock Report by the issue of artificial insemination by husband (AIH) and by donor (AID). The Archbishop of Canterbury in 1948 was highly critical of the practice of AID, though not of AIH, recommending that it should become a criminal offence. But no action was taken.

In 1960, the Feversham Committee, set up by the government to consider AI, reported; it considered that $\mathrm{AIH}$ was an acceptable form of treatment for some couples, but believed that most people within both society and the medical profession were opposed to the practice of AID. It concluded that AID was an undesirable practice, strongly to be discouraged. Since 1960, the practice of AID has continued to grow. In 1968, the then Minister for Health decided that AIH and AID should be available within the NHS if recommended on medical grounds.

I do not wish to discuss whether such a change has been for the better, but the history of attitudes towards AID illustrates how, in the absence of parliamentary restrictions, the views of a progressive minority may become widely adopted. Although the issues involved in in vitro fertilization are not the same, it might be argued that they are of sufficient importance that future changes on such matters as a time limit for experimentation should be subject to parliamentary review. Opinions such as "Why not start with a 14-day limit [on embryo research] imposed by regulation with the intention of relaxing that if the new authority can make a convincing case for doing so, and win public acceptance for its plans?" (Nature 342, $461 ; 1989)$ fuel the fears of those opposed to any advance.

JONATHAN J. EWBANK MRC Laboratory of Molecular Biology, Hills Road, Cambridge CB2 2QH, UK 